\title{
水下传感器网络定位技术发展现状及若干前沿 问题
}

\author{
孙大军，郑翠娥", 崔宏宇，张居成，韩云峰 \\ 哈尔滨工程大学水声工程学院, 哈尔滨 150001 \\ * 通信作者. E-mail: zhengcuie@hrbeu.edu.cn \\ 收稿日期: 2017-11-30; 接受日期: 2018-02-28; 网络出版日期: 2018-08-24 \\ 国家自然科学基金 (批准号: 61531012, 61701132) 和黑龙江省留学回国人员择优资助 (批准号: 2017QD0047) 项目
}

\begin{abstract}
摘要由于海洋环境中温度、压力、波浪、海洋生物等因素的影响, 致使绝大部分陆地无线传感器 网络定位技术无法直接应用于水下传感器网络, 需要结合其独特的海洋声传播环境进行研究. 近十 年来, 水下传感器网络定位技术取得了巨大的进步, 本文综述了水下传感器网络定位技术的研究现 状, 探讨了水下传感器网络定位技术中的若干前沿问题, 并展望了水下传感器节点定位技术在近几 年内预期能够解决的现实问题.
\end{abstract}

关键词 水下传感器网络, 定位, 通信, 网络定位节点, 水下信号传播

\section{1 引言}

近年来, 随着各沿海国家对领海主权的日益重视和海洋资源争夺的日益白化, 以及水下传感器网 络在海洋环境监测、海洋资源开发与利用、地质灾害预报及海洋国防安全等领域的重要应用价值, 水 下传感器网络技术的理论研究及应用得到越来越多的关注. 在水下传感器网络中, 传感器均需关联 相应精度的位置信息来匹配水下传感器网络的任务, 并且在网络协议、协同探测等方面传感器位置信 息亦具有重要价值. 因此, 水下传感器网络定位技术是水下传感器网络应用的基础, 具有重大的研究 意义.

水下动态传感网络主要由自主式水下潜器 (autonomous underwater vehicle, AUV)、遥控无人潜水 器 (remote operated vehicle, ROV)、潜艇等各类潜器组成的动态节点、由各类潜浮标等组成的静态节 点构成. 从水下网络节点定位的角度看, 这些节点又可分为参考节点 (也称针节点) 和普通定位节点, 其区别在于参考节点地理位置已知而普通节点位置未知, 普通定位节点的水下位置需要通过参考节点 的已知地理位置来获得. 水下传感器网络定位技术主要研究如何将位置信息从少数几个参考节点准

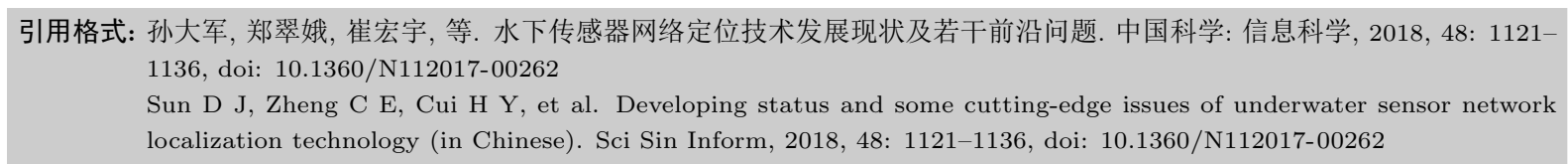


确、可靠、快速地传递到网络中所有的普通节点. 与应用于非海洋环境的普通传感器网络相比, 由于 水下传感器网络以声波为信息载体、以海水为传播介质, 因此其信号传播环境要复杂得多, 水下传感 器网络节点定位具有如下方面特点:

(1) 时变空变的声速. 海水中的声速受温度、盐度、压力等因素的影响, 呈现出时变空变的特点, 声速变化范围在 $1400 \mathrm{~m} / \mathrm{s} \sim 1600 \mathrm{~m} / \mathrm{s}$ 之间, 使得精确距离测量难度大, 而仪器自身误差以及网络长时 间、大范围观测通常会影响通过预测量声速剖面来补偿声线弯曲的效果.

(2) 网络时延高. 声音在海水的传播速度约为 $1500 \mathrm{~m} / \mathrm{s}$, 远低于无线电波在空气中的传播速度, 且 节点间通信距离远 (通常为几百米至几十公里)、通信延迟大; 而水声通信链路的可靠性也远低于无线 电传感器网络, 加剧了网络延迟.

(3) 通信带宽小. 水下无线传感器网络可用带宽严重受限, 通常根据信道的条件可用带宽从几十 赫兹到十几千赫兹不等.

(4) 高精度的地理坐标传递难度大. 由于海洋声信道的复杂性, 通过水面 GPS 向海底传递地理位 置代价大. 通常水下传感器网络希望通过尽可能少的已知针节点位置获得高精度的目标节点位置.

(5) 拓扑结构稀疏. 相对陆地上的无线传感器网络而言, 由于水下布设节点成本高、作业复杂, 水 下传感器网络拓扑结构呈现出稀疏的特性.

(6) 能源有限. 水下节点能源主要依靠电池供给, 不易更换, 而水下节点的声发射耗能远高于陆地 的无线传感器网络节点, 因此在水下传感器网络定位协议设计中, 能源是需要着重考虑的因素.

由于以上水下传感器网络节点定位所面对的挑战, 致使绝大部分陆地无线传感器网络定位技术无 法直接应用于水下传感器网络, 需要结合其独特的海洋声传播环境进行研究. 本文第 2 节综述了水下 传感器网络定位技术的研究现状, 第 3 节讨论了水下传感器网络定位技术中的若干前沿问题, 最后展 望了水下传感器节点定位技术在近几年内能够解决的现实问题.

\section{2 水下传感器网络定位技术发展现状}

水下传感器网络定位技术是由传统的水下声学定位技术与水声通信网络技术相结合发展而来的, 国内外相关研究机构积极开展对水声传感器网络定位的研究, 取得了丰硕的成果. 本节在简要介绍水 声通信网络技术和水下声学定位技术的国内外发展现状基础上, 总结了近年来的比较有代表性的水下 传感器网络定位技术的研究成果.

\section{1 水下通信网络技术发展现状}

尽管水声信道是迄今为止难度最大的无线信道 ${ }^{[1]}$, 但同样水声也是迄今为止唯一可以进行水下远 程信息传输的有效载体. 水声通信技术从最初的低速率 FSK 通信技术 ${ }^{[2,3]}$ 到 20 世纪 90 年代相干通信 技术 ${ }^{[4,5]}$, 产生了跳跃式发展, 随后是对正交频分复用技术 (orthogonal frequency division multiplexing, OFDM $)^{[6,7]}$ 和单载波 (single carrier, SC) 技术 ${ }^{[8 \sim 10]}$ 的研究, 到如今最新的具有强宽容性的非正交 OFDM 通信技术 ${ }^{[11]}$, 面对复杂的水声信道环境, 研究人员在追求最大传输距离和传输速率的道路上 不断地进行探索. 国外在水声通信方面主要研究人员和技术简要汇总如表 $1^{[10,12 ~ 16]}$.

水声通信网络经过二十几年的发展不论是在理论研究还是实验研究上均取得了长足的进步, 有强 烈应用背景的水声通信网络主要有: (1) 美国的 DADS, 为大范围关键海区水下探测的要求而开发, 是 一种通用的分布式水下信息网, 网络由传感器、控制器及武器节点组成, 可以执行水下检测、目标探测 
表 1 研究现状

Table 1 Research status quo

\begin{tabular}{|c|c|c|}
\hline Researchers & Research institute & Research technology \\
\hline Freitag \& Merriam [12] & Woods Hole Oceanographic Institution & MFSK \\
\hline Stojanovic \& Catipovic [13] & Woods Hole Oceanographic Institution & $\begin{array}{l}\text { Coherent communication (DFE based } \\
\text { on embedded PLL) }\end{array}$ \\
\hline Zhou \& Giannakis [14] & University of Minneapolis & OFDM \\
\hline Riedl \& Singer [15] & University of Illinois & $\begin{array}{l}\text { Single carrier (Multi-channel iterative } \\
\text { turbo equalization) }\end{array}$ \\
\hline Edelmann \& Hodgkiss [16] & University of California & $\begin{array}{l}\text { Single carrier (Array-based equalization } \\
\text { technique) }\end{array}$ \\
\hline Zheng \& Xiao [10] & University of Missouri-Columbia & $\begin{array}{l}\text { Single carrier (Equalization technique in } \\
\text { the application of improved linear MMSE } \\
\text { in MIMO communication system) }\end{array}$ \\
\hline
\end{tabular}

及跟踪任务 ${ }^{[17]} ;$ (2) Seaweb, 美国水下网络研究类基础平台, 网络骨干节点由多个自治、静止的传感器 节点和通信中继节点组成, 分别于 2001 至 2006 年间进行了多次海上实验, 可完成海中遥感数据的传 送, 从岸基和船载命令中心向水下平台发送遥控指令 ${ }^{[18]}$; (3) FRONT, 美军水声传感器实验网络, 被 美国海军称作为 Telesonar, 采用水下传感器单元可实现水下二维和三维结构的信息采集及网络传感 器向岸上递送数据或岸上对传感器的遥控指令 ${ }^{[19]}$. 以学术研究及工业应用为目的的国外单位研究内 容主要有: 意大利罗马大学 (Sapienza University of Rome) 及 Padova 大学分别开发了基于 NS-2 平台 的水声通信网络仿真、半实物仿真以及实验控制软件 SUNSET 和 DESERT ${ }^{[20,21]}$; 美国 University of Connecticut 开发的 Aqua-Net ${ }^{[22]}$.

在重要战略价值的埽引下, 我国对水声传感器网络研究也越来越重视, 逐步加大了对水声通信网 络的研究. 中国科学院声学研究所、中国船舶重工集团公司第七一五研究所、中国海洋大学、浙江 大学、厦门大学、哈尔滨工程大学等单位已经开始水声通信网络实验. 中国海洋大学在某实验海域 $500 \mathrm{~m} \times 500 \mathrm{~m}$ 的范围内布放了 20 个浮标形式的传感器节点, 用于采集该区域的光强度、温度等信息, 这些数据可以通过无线模块经过多跳传回到实验室进行分析, 达到实时监测的目的 ${ }^{[23]}$. 中国船舶重工 集团公司第七一五研究所在 “十一五” 期间构建了一个水声通信网实验系统, 2010 年进行了湖上 5 节 点组网实验. 哈尔滨工程大学在 2011 年 10 月于大连王家岛东部海域进行了三节点网络演示实验 ${ }^{[24]}$. 同时, 哈尔滨工程大学基于军事应用需求构建了仿真、半实物仿真以及实验控制一体化的水声通信网 络研究及实验平台, 2014 年 10 月在吉林省松花湖进行了 12 个节点组网实验 ${ }^{[25]}$ (其中水面移动节点 2 个, 水下固定节点 10 个), 并于 2015 年完成了浅海演示网络以及动态组网实验 ${ }^{[26]}$. 另外, 由中国科 学院声学研究所、中国船舶重工集团公司第七一五研究所、哈尔滨工程大学三家单位联合在浙江千岛 湖和南海进行了较大规模的水声组网实验, 网络由 1 个主网关和 3 个子网关组成, 网络总节点数目超 过 10 个, 子网内和子网之间根据数据量自适应选择 ALOHA 和 MACA-W 两种协议, 可以进行子网测 试和联合组网测试 $[27 \sim 29]$.

\section{2 水下声学定位技术发展现状}

水下定位技术始于 20 世纪 50 年代, 主要用于海洋工程、水下营救、海洋科学及军事应用, 是以 


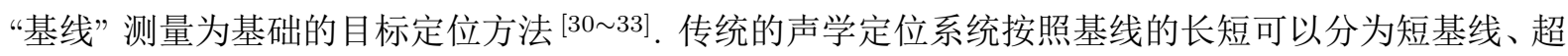
短基线以及长基线, 或者是其中前两者和长基线组合构成的水下综合定位系统 ${ }^{[34 ~ 39]}$. 国内外对水下 定位系统的研究比较成熟, 经过半个多世纪的技术研发及实验应用, 有大量的研究成果及货架产品应 用到实际的海洋工程中. 水下定位技术经历了信号体制由窄带转向宽带、标定方式由单一标定转向结 合多传感器的联合标定及定位解算增加数据融合与滤波算法等阶段, 以追求更高的定位精度、更广的 覆盖范围及更有效的标定方法 [40 42].

国外的水下定位技术发展较早, 目前已经研发出一系列较为成熟的货架产品, 具有代表性的公司 包括英国的 Sonardyne、法国的 IXBLUE 和挪威的 Kongsberg 等. 国内的水下定位技术发展起始于 20 世纪 70 年代末, 经过几十年的努力目前取得了一定的成果. 自哈尔滨工程大学杨士莪院士牵头研发 洲际弹道导弹落点测量系统以来, 哈尔滨工程大学、中国科学院声学研究所和中国船舶重工集团公司 第七一五研究所等少数机构从事了水声定位系统的研制 [43]. 自 “十一五” 以来, 水声定位技术在国内 得到长足的发展, 中国测绘科学院与中国船舶重工集团公司第七一五研究所共同研制的 “水下 DGPS 高精度定位系统” 能够为水下航行体提供精确的定位和授时, 哈尔滨工程大学十余年来逐步发展超短 基线定位技术和长基线定位技术, 其研究的超短基线定位系统已在多条科考船上安装, 研制的长基线 定位系统也为各类潜器提供水下高精度定位服务. 这是继美国、法国等发达国家率先开展水下定位跟 踪技术研究后, 我国在该研究方向取得的重大研究成果 ${ }^{[44 ~ 47]}$.

\section{3 水下传感器网络定位技术发展现状}

随着关于水声通信和水声通信网络技术的研究不断取得进步, 围绕水下传感器网络节点数据采 集位置关联 ${ }^{[48,49]}$ 、网络节点协同探测数据融合 $[50,51]$ 、移动节点导航定位校准 ${ }^{[52]}$ 以及网络协议优 化 ${ }^{[53} 55$ ] 等需求, 国内外学者开展了多种多样的水下网络节点定位技术的研究. 与传感器网络通信技 术、传统的水下定位技术已经处于大规模的实验与应用阶段不同, 水下传感器网络定位的研究还处于 基础阶段, 近几年来才有少量的湖上及海上实验验证. 近年来比较具有代表性的研究成果如下.

(1) 单节点定位技术. 由于水下声信道的复杂性, 许多无线传感器网络广为应用的定位技术在移 植到水下时遇到了困难. 文献 [56] 提出了基于样本匹配的定位方法, 通过在待定位区域内目标可能出 现的位置离线状态采集样本, 在线将接收到的信号与离线样本进行对比, 通过模式匹配、概率映射或 主分量分析的方法估计目标的位置, 其定位精度取决于特定区域内离线状态样本的数量, 不适用于外 场的时变水声环境. 文献 [57] 提出了区域定位方法, 参考节点以不同功率发射信号, 利用声传播衰减 模型划分待定位区域, 根据接收信号的强度来确定目标所处的区域, 该方法的平均定位精度为 0.75 倍 距离.

由以上定位模型可以看出, 在无线传感器网络中常用的基于样本匹配的方法及区域定位方法定位 在水下传感器网络中的应用难度大. 因此, 水下传感器网络定位中以几何测量原理为主, 参考节点与 定位节点的距离在单节点通信范围之内时, 直接测量距离并交汇定位. 其方法主要以基于接收信号强 度 (received signal strength indication, RSSI) 和信道模型联合修正 ${ }^{[58]}$ 方法和基于时间的测量方法为 主 (ToA、TDoA), 其中前者由于水声信道的复杂性, 其定位精度不高; 后者能够很好地保证精度, 但通 信开销过大. 总体说来基于距离测量的、有精度能力保障的几何测量原理仍是研究的主流.

在基于时间的测量方法中, 文献 [59] 提出一种被动式免同步的水下传感器网络定位技术 (underwater positioning scheme, UPS), 参考节点按先后顺序发送定位信息, 待定位节点只是被动接收参考节 点发送的定位信息通过 TDOA 测距实现静默定位, 具有较低的通信消耗. 但是对针节点的合作能力要 求较高, 若有针节点信息接收失败则很难定位. 文献 [60] 针对 UPS 中存在单个时延解算失败导致定 
位不成功的问题, 通过增加时间溢出机制提高了定位成功率. 文献 [61] 等结合 UPS 与 LBL, 提出一 种异步定位方法, 对定位发起节点采用应答式测距, 其他待定位节点采用 UPS, 适用于稀疏网络定位, 并分析了数据包丢失对性能的影响, 文献 [62] 通过深度传感器辅助将针节点坐标投影至目标节点水平 面, 即三维水声定位问题就转换成二维定位问题, 被定位节点利用三维 $\mathrm{TOA}$ 测距结果在水平面的投 影进行定位, 该非退化的投影不影响网络的可定位性.

(2) 大规模网络、高覆盖率的定位技术. 在大规模网络、高覆盖率的定位技术方面, 主要有通过增 加移动节点来提高定位覆盖范围, 以及通过将被定位的普通节点升级成针节点实现多跳网络的逐级定 位两类技术手段.

文献 [63 65] 等提出和利用能够垂直方向移动信标辅助定位方法 (localization with dive and rise beacons), 引入可垂直运动的信标, 通过与水面浮标的 GPS 通信获得位置再在不同深度上广播位置来 减少通信距离, 提高定位覆盖率. 为了减少垂直信标的升潜次数并加速信标的运动速度, 文献 [66] 用 AUV 代替了垂直信标, AUV 通过周期性的浮出水面与 GPS 通信获取自身坐标后下潜, 以航位推算方 式更新位置并广播. 中国海洋大学等在 “海洋感知” (OceanSense) 的项目中提出垂直相交的定位技术, 以 AUV 作为移动的参考节点, 联合普通节点和参考节点运动轨迹的几何关系提升了 RSSI 测距精度、 降低了系统开销、优化了参考节点运动轨迹, 并进行多次外场实验验证 $[67,68]$.

文献 [69] 提出一种应答式测距的传感器网络节点三维定位技术, 并通过将被定位的普通节点升级 成针节点实现多跳网络的逐级定位. 为了控制多跳网络逐级定位的误差. 文献 [70] 等通过设定被定位 普通节点升级成针节点并联合逐级迭代算法和扩展欧几里德方法提高网络定位的覆盖率, 适合于大规 模网络定位. 基于多跳网络, 文献 [71] 利用 2 跳范围内的针节点协同定位, 由未知节点定位、迭代定 位、改进的三维欧几里得测距和三维 DV 距离估计 4 个步骤完成,仿真证明了该方法提高了定位覆盖 率与精度并降低了能耗. 文献 [72] 提出一种距离相关的定位技术, 该技术将测距过程按照物理层符号 速率的不同分为两种模式, 以完成单参考节点下的定位, 该种方法可以消除多跳网络定位的累积误差. 文献 [73] 针对孤立节点提出了一种基于多跳的定位方法, 通过寻找针节点与待定位节点之间的最短路 径并求其对应直线距离来交汇解算目标节点的位置.

(3) 系统误差修正技术. 为了实现高精度的水下传感器网络定位, 系统误差修正必不可免, 目前的 研究主要体现在节点动态特性补偿及声速误差修正技术方面.

文献 $[74,75]$ 等针对新节点加入网络的情况提出一种基于交替迭代计算的联合时间同步节点定位 方法, 并通过射线跟踪以及 IMM 滤波器补偿了声线弯曲和节点动态性, 提高了定位精度, 有效地降低 了通信开销. 针对声波在海水中的传播速度慢给漂移节点带来的节点收发位移大的问题, 文献 [76] 通 过设定时间窗优选有效双程往返时间来提高观测数据的有效性, 并通过添加声速为待求未知量的方式 提升了在不确定声速下的节点定位精度.

针对移动目标, 文献 $[77,78]$ 等提出一种基于因子图的极大似然离线集中式定位方法实现移动目 标轨迹的极大似然估计; 并提出一种联合同步的高精度定位方法, 在速度信息的辅助下, 利用单向测 距信息对位置和时间偏差进行联合估计, 海上实验验证了以上方法的有效性.

文献 $[79,80]$ 等提出一种基于移动节点的联合网络同步的定位方法, 静态节点通过接收一个已知 位置移动节点周期性广播的定位包来实现同步与定位, 并分析了定位与同步的成功概率. 针对声速未 知条件下联合网络同步的定位, 提出先通过针节点之间的通信来求解声速, 再利用 ToA 进行节点同步 与定位.

(4) 定位协议. 在定位协议方面: Leus 等 ${ }^{[81]}$ 和 Stojanovic 等 ${ }^{[82]}$ 研究了在针节点均为具有无线 电通信功能的水面浮标的情况下对水下节点定位协议, 给出了理论的最小定位时间, 分析了协议的耗 
能、定位精度等性能. Zhang 等 $[83]$ 针对深水作业条件提出了一种多跳的反向定位机制, 通过深度信息 控制路由, 同步求解了定位与路由问题, 在浅水性能不损失的条件下提升了深水性能. Liu 等 ${ }^{[84]}$ 提出 基于 TDOA 的大规模水下网络定位技术, 该技术基于反向多跳测距方法, 由水面船对水下潜航器进行 定位跟踪. Han 等 ${ }^{[85]}$ 分析声速剖面变化等因素对测距误差以及网络定位性能的影响. Guo 等 ${ }^{[86]}$ 提 出垂直相交的定位技术, 该技术采用一个移动的参考节点进行定位, 既利用基于 RSSI 的测距方法, 又 利用普通节点和参考节点运动轨迹的几何关系, 提升了 RSSI 测距精度, 降低了系统开销.

(5) 网络定位性能评价. 有关网络定位性能的评价研究也是目前水下传感器网络定位研究的热点 问题之一. 2002 年文献 [38] 讨论了水声定位有关的科学问题, 重点提出 5 种评价网络定位的准则, 即 定位精度、定位速度以及定位节点覆盖率、通信开销以及网络规模影响等问题, 至今仍是评价网络定 位性能的主要方面. 目前的研究尚属初步, 特别是对于定位精度的讨论和网络规模影响的讨论涉及很 少. 网络定位的精度涉及网络最终的用途是否广泛, 需要重点解决的问题是, 网络定位能否达到或接 近达到传统定位系统的精度效果, 特别是在深海; 而网络规模影响涉及作业效率和通信开销等更多方 面, 需要重点解决的问题是, 定位节点如何能够以尽可能安静的方式 (不发声音或将信息传递最少, 有 时称为被动定位) 实现定位, 这样网络规模才能提升, 网络整体性能才能提高.

此外, 目前水下传感器网络性能的评价方面研究, 大多还是数值模拟为主, 近几年才出现了少量的 湖上与海上实验分析.

\section{4 结论与发展趋势分析}

从以上情况介绍可以看出, 目前国内外对水声通信和通信网络的研究与实践已达到相当水平, 能 够实现网络节点信息的互联互通, 这是水下网络节点定位研究的重要基础. 从水下网络定位技术自身 发展来看, 仍有如下几方面的发展趋势需要予以关注.

(1) AUV 配备的传感器种类与水平越来越高, 水下作业向精细化发展趋势明显, 要求单节点定位 精度、协同探测定位精度也越来越高. 目前有关水下网络定位精度的研究尚缺乏系统性, 特别是与海 洋信道、平台运动性以及网络协议影响密切相关的水下网络定位精度模型缺乏深入研究.

(2) 水下 AUV 群作业模式越来越受到重视, 如何依赖水声网络技术实现网络内大规模节点的位 置确定, 对水下网络定位技术提出强烈挑战, 精确快速的网络定位算法与相适应的网络协议需求强烈.

(3) 对定位性能的评价研究不够, 包括定位精度、可靠有效定位覆盖和水声网络中可定位性等问 题的研究, 既决定了水下传感器网络定位技术的适用范围, 也是定位算法及协议设计的重要标准.

(4) 对网络定位的参考节点定位方法与性能的研究没有引起足够的重视, 这是提升网络定位精度 性能的关键所在, 特别是围绕深海高精度网络定位的需求.

(5) 目前网络定位的实验研究尚缺乏评估技术指导下的系统性, 多种网络定位协议与算法也缺乏 实验的检验.

\section{3 水下传感器网络定位技术若干前沿问题}

\section{1 水下动态节点网络定位基础理论体系研究}

面对水下传感器网络的特殊性, 需要系统建立融合复杂海洋环境、平台运动特性以及网络自身特 性为一体的水下网络定位理论模型和信号处理方法的模型, 主要包含以下 4 个方面的内容. 


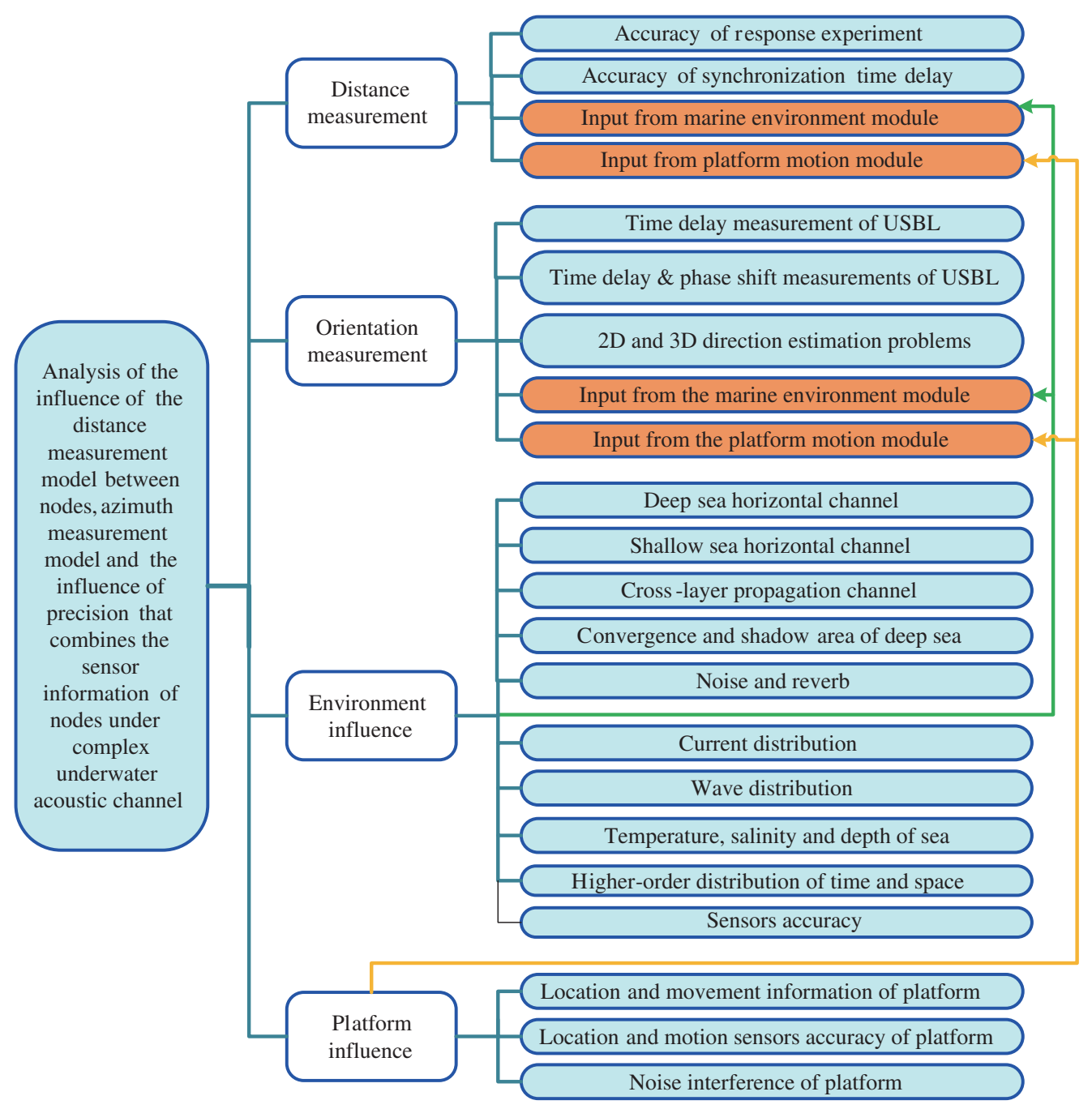

图 1 (网络版彩图) 影响传感器节点距离及方位测量精度的因素

Figure 1 (Color online) Factors affecting sensor node distance and azimuth measurement accuracy

(1) 水声复杂信道条件下结合节点自身传感器信息的水下节点间距离与方位测量模型与精度影响 分析. 水声信道的复杂性是建立水下传感网络最大的障碍, 影响水声通信、探测以及定位性能等多个 方面. 对于追求高精度为目标的水下网络定位而言, 更需要结合海洋环境、平台运动特性和节点自身 特性进行综合建模, 充分认识理解影响定位精度的各种因素, 并在节点直接观测量 (如时延) 基础上进 行模型修正, 精确获得各种参数. 在影响水下网络节点定位的众多因素中, 节点间的距离测量和方位 测量是网络定位的基础和根本, 应对如图 1 所示因素进行系统建模, 分析各种因素在多种不同情况的 影响.

(2) 水下参考节点位置与时间信息获取方法, 如图 2 所示. 水下参考节点的位置对于高精度水下 网络定位十分重要, 特别是在深海应用场合更为重要, 但在公开文献中参考节点位置几乎假设为已知 或讨论很少. 此外, 水下参考节点的布放和回收作业相对复杂 (特别是深海), 参考节点的时钟信息随 着时间的增长, 时钟偏差和时钟斜率都会与参考时钟 (如 GPS 时钟) 不同. 这方面公开文献有许多讨 论, 但基于水下高精度距离测量的时钟同步性能和授时协议等因素对定位精度的影响尚需系统分析. 


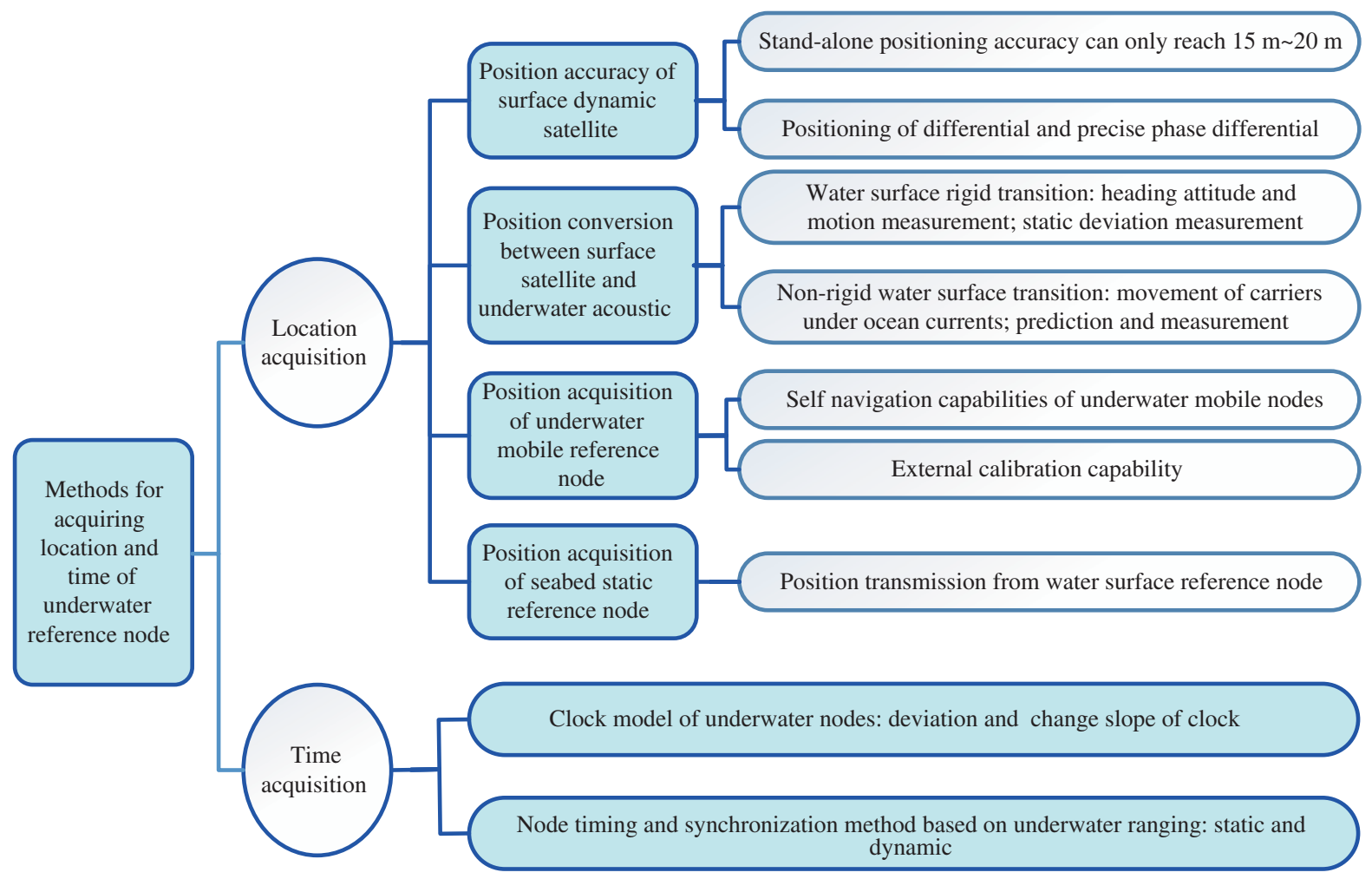

图 2 (网络版彩图) 水下参考节点位置及时间获取方法

Figure 2 (Color online) Underwater reference node location and time acquisition method

(3) 水下网络定位信号建模、检测、估计、跟踪和融合方法. 水声频带可利用信息比无线电少得 多. 如何结合水声复杂信道特性和有效的频率资源, 借鉴无线通信中多用户通信理论基础进行水声定 位信号建模, 并在实际水声信道条件下开展信号的稳健检测、参数高精度估计、信号的稳定跟踪和信 息高效融合是水下网络定位成功与否以及性能好坏的关键因素. 目前研究尚不系统, 研究思路上, 首先 是考虑稳态信道、随机信道、时变特性、环境与平台干扰和多用户干扰的复杂信道模型的统计传输函 数建立, 然后是多用户干扰环境下结合水声通信技术的稳健信号检测算法和高精度参数估计算法, 具 体包括基于水声信道估计的稳健相干检测和基于水声通信多普勒序列精细估计技术和补偿技术, 最后 是结合平台信息的多用户数据融合模型与跟踪算法, 典型的有单节点平台运动信息融合节点轨迹和有 限数据信息支持下多用户轨迹跟踪与数据融合.

(4) 网络协议层算法对水下动态节点定位精度影响的理论分析. 水下信息网络从物理层和协议层 两个方面解决了水下节点间信息链路的建立和节点间互联互通问题. 信息的重传和路由的重建等问题 对于信息互联的影响主要表现在网络延迟问题,但对网络定位而言, 网络协议层算法的大时延和不确 定性对网络定位的影响是致命的. 目前尚缺乏系统性的研究, 利用通信协议与时间关系建立不确定的 因素分析，建立包括身份、位置及精度、时间及精度、运动及精度、路由、任务的水下网络节点定位的 协议, 分析大时延航行器动态节点同步协议与性能.

\section{2 大规模水下动态网络精确快速网络定位技术}

该技术主要解决的问题为 AUV 集群大范围水下作业时如何进行高精度节点定位. 通常水下主 


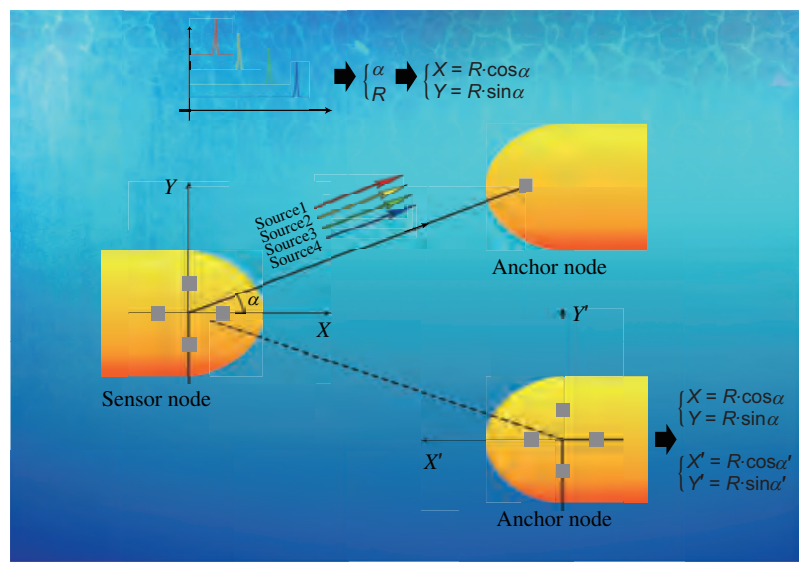

图 3 工作原理示意

Figure 3 Principle of the work

AUV 节点通过配备导航设备 (如惯导等) 具有一定精度的自主导航能力, 这样参考节点 AUV 可以提 供水下绝对地理坐标 (惯导推算或者到水面通过 GPS 对惯导漂移偏差进行校准后惯导推算).

对水下传感网络定位性能影响较大的是网络节点的规模, 因为传统网络定位需要通过多个节点间 相互测距来确定节点的位置. 当节点数目增加时, 网络通信开销几乎呈几何级数增长. 因此必须寻求 适合大规模水下动态网络节点定位的新方法. 目前公开文献的研究仅限在海底有多个参考节点支持时 的情况. 但没有海底参考节点参与时, 如何实现大规模大范围水下动态网络节点定位尚没有有效解决 办法.

基于动态参考节点多发单收方位测量 (MISO-AOA) 或多发多收方位测量 (MIMO-AOA) 的技术 体制可解决此问题, 实现单跳通信范围内允许的全部定位节点定位. 即在参考节点 AUV 上布置与惯 导参考系一致的声学基阵 (如果不一致, 安装偏差需要事先校准), 该基阵由具备 $N$ 只 (3 只以上) 的 高精度位置关系的声源构成, 能够在网络协议的引导下定期或被请求广播定位信号帧. 定位信号帧主 要包含参考节点发射源的身份识别信息、发射时刻、位置、航向姿态、深度及各参数的精度水平等信 息. 定位节点安装单只接收传感器就可根据 $N$ 通道定位信号帧的信号处理获得接收传感器的地理坐 标. 基本原理参见图 3.

定位节点上多只接收传感器组成接收基阵的目的是能够使得定位节点也能够以被动方式确定参 考节点的位置, 可进一步校核节点定位可靠性, 提高一定的定位精度的同时, 又可作为二级参考节点 (精度略差) 对外围定位节点提供节点定位服务.

上述原理能够使得定位节点以被动的方式 (不发声音) 实现高精度定位. 但定位精度仍然受到复 杂水声信道、平台运动特性、节点间时间同步性以及定位协议本身多种因素的影响. 需要系统分析其 优缺点和实际使用限制, 特别是定位精度模型与误差修正办法, 需要予以特别关注. 此外参考节点采 用 MISO/MIMO - AOA 体制获得位置信息的定位节点可以作为二级参考节点 (其位置精度等级为二 级), 对外提供网络定位服务. 不同网络拓扑结构的这些误差传递规律和混合精度等级的节点定位协议 需要进行充分研究. 在此过程中, 结合部分先验信息的网络定位协议和网络性能需要进行深入的分析.

\section{3 基于海底参考节点的免同步水下网络定位技术研究}

(1) 水下网络定位技术工作原理. 基于海底参考节点可以获得水下动态节点的位置, 甚至是无需 


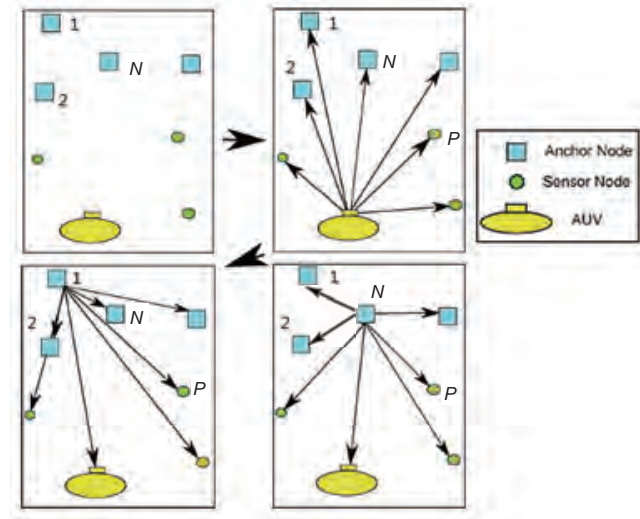

(a)

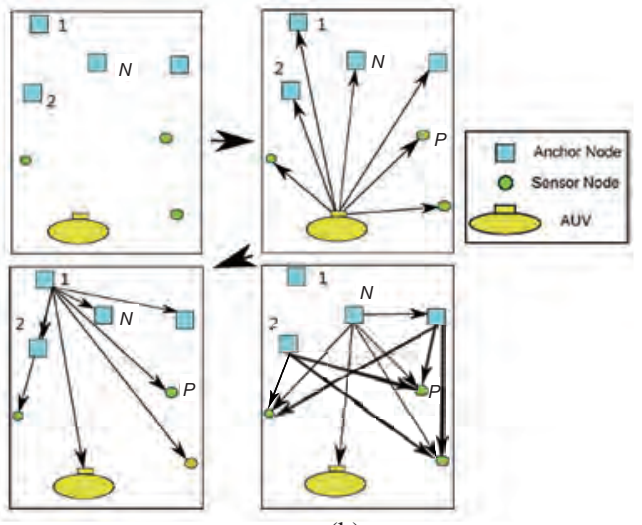

(b)

图 4 (网络版彩图) 免同步水下网络定位原理. (a) Cheng 和 Zhou 方案; (b) 改进方案

Figure 4 (Color online) Avoid synchronous underwater network positioning principle. (a) Cheng and Zhou programs; (b) improvement programs

海底参考点同步, Cheng ${ }^{[59]}$ 和 Zhou ${ }^{[64]}$ 对该方案进行了理论分析和湖上实验验证. 其定位协议 (如 图 4 所示) 如下: (i) 由网络定位发起者 AUV 启动定位命令, 图中方块形的参考节点和定位节点均收 到此命令, 例如参考节点 1 到 $N$, 定位节点 $P$; (ii) 按照协议约定的次序, 1 号参考节点先应答, 此时定 位节点 $P$ 收到 1 号的应答信号; (iii) 接着 2 号参考节点再应答同一个询问命令, 为了防止协议碰撞, 1 号和 2 号应答间隔保护时间; (iv) 以此类推, 最后 $N$ 号参考节点的应答信号被定位节点 $P$ 收到之 后, 定位节点将具有一系列节点间距离信息, 由此定位节点 $P$ 的位置可以由自身以不发声的被动方式 解算, 而 AUV 也可获得自身的位置.

从上述模型工作过程可以看出, 整个定位协议的时间很长, 当追求水下高精度网络定位能力时, 该 方案采用的每个海底参考节点 “轮询” 的定位协议算法, 效率低, 只能应用于小规模的水下网络. 并且 效率低下的定位协议算法导致定位作业时间长, 期间节点的运动性会带来额外的定位误差. 但对定位 节点 $P$ 来说, 其非同步被动定位的优点在大规模水下节点定位中具有显著的优势. 若动态节点采用多 用户工作体制的方法, 则可减少原有模型中的网络通信开销, 提高网络定位速度; 同时降低节点运动 性的影响, 提高网络定位精度. 最重要的, 定位节点也以被动 (不发声) 非同步的方式工作, 适合大规模 网络节点定位.

(2) 海底参考节点高精度获取方法. 影响上述网络定位精度的另外一个因素是如何提高海底参考 节点的位置精度, 这个问题在深海将显得尤为重要. 这个问题本质上是如何将海面获得的卫星定位结 果高精度传递到水下. 目前, 公开文献上关于此方面的研究讨论很少.

在现行的海洋监测和科学考察中通常是通过装配有 GPS 的水面船只对待定位海底节点绕行, 在 绕行过程中不断通过声通信获取水面船与待定位节点的距离, 并通过空间距离交汇原理获得, 实现水 面位置向水下位置传递. 水面船只节点与海底节点间信息交换是通过声波进行传递的. 由于海水的温 盐深特性 (决定了海水的声速深度剖面是弯曲的) 是水平分层的, 如图 5 所示, 声波传播路线是弯曲 的, 这是造成海底参考节点位置确定不准确的主要原因. 注意到海洋声速是水平分层的特性 (即同一 深度的声速差别远比不同深度的声速差别要小很多), 本研究利用这一特性通过水面船只航迹控制和 数据处理的方法, 消除信道引起的对称性测距误差, 提升海底参考点的定位精度.

以二维定位为例说明 (如图 6 所示), $T 1$ 和 $T 2$ 是两个水面船只位置, $T 0$ 是海底参考节点的位置. 尽管 $T 1$ 和 $T 2$ 均有 GPS 测量误差的存在, 由于对称性, 声线弯曲引起的参考节点的水平位置估计与 


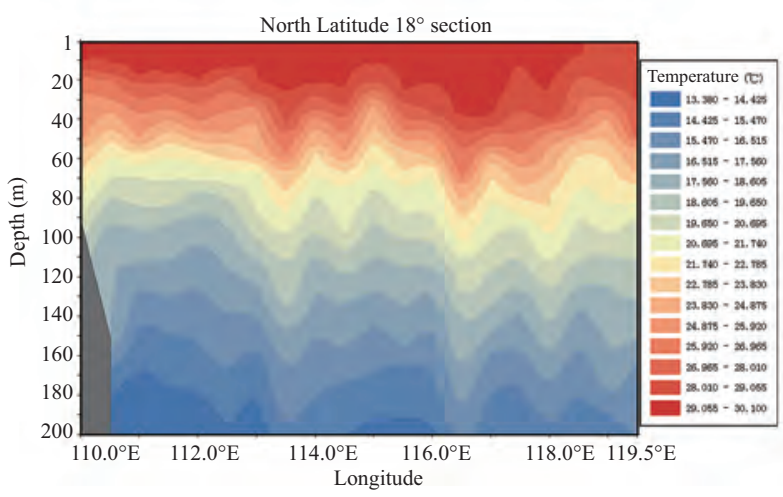

图 5 不同区域温度与深度变化情况

Figure 5 Variation of temperature and depth in different regions

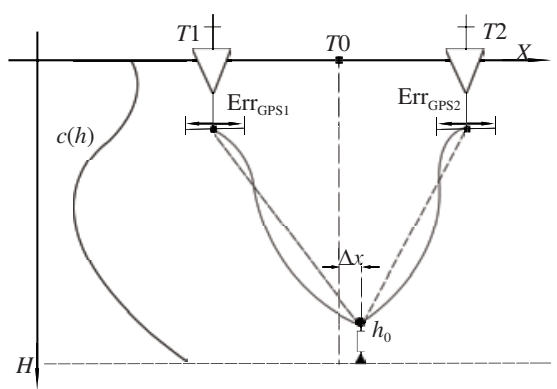

图 6 二维定位基本原理

Figure 6 Two-dimensional positioning of the basic principles

弯曲弱相关, 从而水平坐标可精确被确定, 大大放宽对声速误差的时延补偿要求. 实际海上操作和数 据处理要复杂多. 采用该原理, 即便在几千米的深海, 海底参考节点的位置精度也能够保持在米量级 的高精度水平.

(3) 动态节点位置高精度获取、误差模型与综合修正方法. 上述原理能够在动态节点被动工作的 情况下获得自身位置. 其精度取决于许多因素, 特别是海洋环境因素、平台运动特性以及节点自身信 号处理算法. 需要系统分析该方法的优缺点和实际使用限制因素, 特别关注海底参考节点位置校准精 度对节点定位精度的影响等方面. 同时, 还要根据先验信息和现场获得信息进行综合误差的修正, 进 一步提升网络定位性能.

\section{4 水下传感器网络定位技术实验平台建立与实验验证}

水下网络定位性能一般从定位精度、定位效率、定位覆盖率、通信开销以及网络规模影响等几个 方面进行评价. 到目前为止, 水下传感器网络定位技术研究以理论研究为主, 定位技术的性能分析及 验证多是通过计算机仿真进行, 在近几年才出现有少量的湖上、海上实验验证. 而实际上, 由于海洋环 境的复杂性, 计算机仿真很难全面准确地表达传感器网络定位技术的实用性能, 需要在实际的海洋环 境中测试分析, 因此需要尽快建立水下传感器网络定位技术验证平台. 目前国内的水下传感器网络技 术的实践表明从网络通信的角度已经具备了建立水下传感器网络定位技术验证平台的基础.

考虑到浅海与深海之间的巨大环境差异及应用需求差异, 建议验证平台的分别建立. 在开展关键 技术问题的海上验证实验时需着重考察两个重点, 一个是考察不同信道条件对网络定位性能的影响, 一个是考察平台运动性对网络定位性能影响实验.

\section{4 总结与展望}

海洋事关国家安全、资源开发和科学探索重大战略利益, 十九大报告指出 “加快建设海洋强国”. 水下传感器网络则是认知海洋、经略海洋的利器, 而水下传感器网络定位则是有效认知海洋的技术基 础, 具有广阔的应用前景. 与陆地上较为成熟的无线传感器网络定位技术相比, 水下传感器网络定位 必须克服水声信道环境复杂、水声平台干扰严重和系统实现困难等挑战. 水下传感网络定位技术已经 
得到大量的深入研究, 已经由基础研究进入到海上实验分析阶段, 即将迈入大规模海上测试与尝试性 应用实践阶段, 可预期节点定位技术的进步将在近几年内获得如下几方面的现实意义.

(1) 动态节点可依赖其位置信息获得自身传感器信息的位置关联处理能力. 海洋动态节点根据作 业任务的不同配备了诸如旁扫声纳、前视声纳、多波束声纳、浅剖声纳、重力计、磁力计、生物传感 器、化学传感器以及各类军用传感器. 所有传感器均需要对获取的传感数据进行不同定位精度的位 置关联处理. 举例来说, 水下潜器高精度海底测绘采用的最新合成孔径声纳 (synthetic aperture sonar, $\mathrm{SAS}$ ) 成像能够达到 $500 \mathrm{~m}$ 距离上像素分辨单元优于 $5 \mathrm{~cm}$, 如果无法匹配足够精度的定位信息, 获得 的高分辨声学图像价值将大打折扣.

(2) 节点可依赖其位置信息完成更高级节点的传感器信息融合和协同处理, 对于数据深度挖掘意 义重大. 尽管水下各类潜器的能力不断提升, 但单平台的感知和处理能力始终有极限. 更何况考虑综合 费效比因素, 单平台的在线处理能力将会受到限制. 水下动态组网的优势在于依托水下可靠、高速的 网络能力进行分布式网络节点信息的收集和汇总, 在更高层面网络节点进行在线甚至是离线处理, 深 度挖掘数据信息. 这时, 各个节点的位置信息以及对应的时间信息对于多节点协同探测至关重要. 节点 定位技术将为基于网络的协同探测奠定重要基础.

(3) 动态节点可依赖其位置信息完成自身的导航系统参数的校准作业. 动态节点一般都配备自身 的导航系统, 主要是有 3 种自主的导航手段: 航向姿态传感器 + 转速信息进行速度推算; 航向姿态传 感器 + 声学对海底/对水层速度推算; 惯导 + 声学对海底/对水层速度推算. 尽管这几种自主的导航 方式成本代价与性能千差万别, 但有一个共同的特点, 即随着时间的增大, 积累导航误差会持续增大, 总是会需要上浮水面进行卫星定位校准或者因为导航偏差达到难以容忍的程度而放弃作业任务. 水下 动态传感网络节点自身位置的获得技术可以将动态节点的外测位置信息通过水下信息链路的方式注 入给相应的动态节点, 校准修正导航偏差, 提升节点以及整个网络的导航能力, 从而提升网络的总体 作业能力.

(4) 已知网络节点位置将有利于降低网络物理层干扰和网络协议层冲突风险, 便于优化网络层路 由协议设计, 提升网络整体性能. 水声信道的复杂性是水下传感网络建设的难点所在, 不仅反映在物理 层上可用带宽有限、信道衰落起伏严重, 还体现在物理层上节点间传输时延大, 这将导致物理层多用 户干扰和协议层介质征占 (medium access control, MAC) 冲突. 如果网络协议层设计能够具有充分、 及时和精确的节点位置信息, 那么网络协议层有可能通过时间控制等信息进行协议优化, 降低冲突风 险, 更能充分发挥物理层的数据传输效率. 自组织网未来必将成为水下动态传感网络的主要形式. 失 效节点的路由剔除和新加入节点的路由建立, 将因为获得的网络节点位置信息, 可以最高效率地被完 成, 网络性能将由此得到极大提高.

\section{参考文献}

1 Rappaport T. Wireless Communications. Upper Saddle River: Prentice Hall, 1996

2 Catipovic J, Deffenbaugh M, Freitag L, et al. An acoustic telemetry system for deep ocean mooring data acquisition and control. In: Proceedings of OCEANS, Seattle, 1989. 3: 887-892

3 Merriam F, Frey D, Catipovic J. Performance of an MFSK acoustic telemetry system. In: Proceedings of OCEANS, Newport, 1992. 2: 928

4 Stojanovic M, Catipovic J, Proakis J G. Adaptive multichannel combining and equalization for underwater acoustic communications. J Acoust Soc Am, 1993, 94: 1621-1631

5 Stojanovic M, Catipovic J A, Proakis J G. Phase-coherent digital communications for underwater acoustic channels. IEEE J Ocean Eng, 1994, 19: 100-111 
6 Li B S, Zhou S L, Stojanovic M, et al. Pilot-tone based ZP-OFDM demodulation for an underwater acoustic channel. In: Proceedings of OCEANS, Boston, 2006. 1-5

7 Li B S, Huang J, Zhou S L, et al. MIMO-OFDM for high rate underwater acoustic communications. IEEE J Oce Eng, 2009, 34: 635-644

8 Choi J W, Riedl T J, Kim K, et al. Adaptive linear turbo equalization over doubly selective channels. IEEE J Ocean Eng, 2011, 36: 473-489

9 Song H C, Hodgkiss W S, Kuperman W A, et al. Spatial diversity in passive time reversal communications. J Acoust Soc Am, 2006, 120: 2067-2076

10 Tao J, Zheng Y R, Xiao C, et al. Robust MIMO underwater acoustic communications using turbo block decisionfeedback equalization. IEEE J Ocean Eng, 2010, 35: 948-960

11 Hoang T D, Le L B, Le-Ngoc T. Joint sub-channel and power allocation for D2D communications for celluar networks. In: Proceedings of 2014 IEEE Wireless Communications and Networking Conference (WCNC), Istanbul, 2014. 13381343

12 Freitag L E, Merriam J, Frye D, et al. A long-term deep-water acoustic telemetry experiment. In: Proceedings of OCEANS, Hawaii, 1991. 1: 254-260

13 Stojanovic M, Catipovic J, Proakis J G. An algorithm for multichannel coherent digital communication over long range underwater acoustic telemetry channels. In: Proceedings of OCEANS, Newport, 1992. 2: 577-582

14 Zhou S 1, Giannakis G B. Finite-alphabet based channel estimation for OFDM and related multicarrier systems. IEEE Trans Commun, 2015, 49: 1402-1414

15 Riedl T, Singer A. MUST-READ: multichannel sample-by-sample turbo resampling equalization and decoding. In: Proceedings of OCEANS, Bergen, 2013. 1-5

16 Edelmann G F, Hodgkiss W S, Kim S, et al. Underwater acoustic communication using time reversal. In: Proceedings of OCEANS, Honolulu, 2001. 4: 2231-2235

17 Roy T N. Deployable autonomous distributed system: future naval capability in undersea warfare. SSC San Diego Biennial Rev, 2003, 3: 181-185

18 Rice J A. Enabling undersea forceNet with seaweb acoustic networks. In: Proceedings of the 5th International Symposium on Technology and the Mine Problem, Monterey, 2002. 174-180

19 Rice J A. Seaweb network for FRONT oceanographic sensors. San Diego: Space and Naval Warfare Systems Center, 2002. 30-35

20 Petrioli C, Petroccia R, Potter J, et al. The SUNSET framework for simulation, emulation and at-sea testing of underwater wireless sensor networks. Els Ad Hoc Net Phys Com, 2015, 34: 224-238

21 Toso G, Masiero R, Casari P, et al. Field experiments for dynamic source routing: S2C EvoLogics modems run the SUN protocol using the DESERT underwater libraries. In: Proceedings of OCEANS, Hampton Roads, 2012. 1-10

22 Peng Z, Zhou Z, Cui J H, et al. Aqua-Net: an underwater sensor network architecture: design, implementation, and initial testing. In: Proceedings of MTS/IEEE OCEANS, Biloxi, 2009. 1-8

23 Wang Y, Liu Y, Guo Z. Three-dimensional ocean sensor networks: a survey. J Ocean Univ China, 2012, 11: 436-450

24 Qiao G, Liu S, Sun Z, et al. Full-duplex, multi-user and parameter reconfigurable underwater acoustic communication modem. In: Proceedings of OCEANS, San Diego, 2014. 1-8

25 汪生泉. 水声传感器网络 MAC 层协议研究. 哈尔滨: 哈尔滨工程大学, 2015

26 Ma L, Qiao G, Liu S. HEU OFDM-modem for underwater acoustic communication and networking. In: Proceedings of the International Conference on Underwater Networks \& Systems, Rome, 2014. 12-14

27 Liu S, Ma L, Hui L, et al. Design and implementation of OFDM underwater acoustic communication algorithm based on OMAP-L138. In: Proceedings of International Conference Underwater Networks \& Systems, Rome, 2014. 12

28 Liu S, Ma T, Qiao G, et al. Biologically inspired covert underwater acoustic communication by mimicking dolphin whistles. Appl Acoust, 2017, 120: 120-128

29 Qiao G, Zhao Y, Liu S, et al. Dolphin sounds-inspired covert underwater acoustic communication and micro-modem. Sensors, 2017, 17: 2447

30 Li S J, Bao G S, Wu S G. A practical overview and prospect of acoustic positioning technology. Ocean Tech, 2005, 24: 130-135 [李守军, 包更生, 吴水根. 水声定位技术的发展现状与展望. 海洋技术学报, 2005, 24: 130-135]

31 孙思萍. 水声定位技术的应用. 海洋技术学报, 1993, 4: 31-34 
32 Sun D J, Zheng C E, Qian H B, et al. The application of underwater acoustic positioning systems in ocean engineering. Tech Acoust, 2012, 31: 125-132 [孙大军, 郑翠娥, 钱洪宝, 等. 水声定位系统在海洋工程中的应用. 声学技术, 2012, 31: $125-132]$

33 Sun D J, Zheng C E. Study on the development trend of underwater acoustic navigation and positioning technologies. J Ocean Tech, 2015, 34: 64-68 [孙大军, 郑翠娥. 水声导航、定位技术发展趋势探讨. 海洋技术学报, 2015, 34: 64-68]

34 Vickery K. Acoustic positioning systems. A practical overview of current systems. In: Proceedings of the 1998 Workshop on Autonomous Underwater Vehicles, Cambridge, 1998. 5-17

35 Vickery K. Acoustic positioning systems. New concepts-the future. In: Proceedings of the 1998 Workshop on Autonomous Underwater Vehicles, Cambridge, 1998. 103-110

36 张光亮, 方世良, 徐步安. 单频脉冲水声信号的精密时延估计研究. 见: 2008 年全国声学学术会议, 2008

37 Coates R F W. Underwater acoustic systems. London: Macmillan Education, 1990

38 Burdic W S. Underwater acoustic system analysis. J Acoust Soc Am, 1984, 76: 996

39 Thomson D, Elson S. New generation acoustic positioning systems. In: Prcoeedings of OCEANS, Biloxi, 2002. 3: $1312-1318$

40 Benslimane A, Saad C, Konig J, et al. Cooperative localization techniques for wireless sensor networks: free, signal and angle based techniques. Wirel Commun Mobile Comput, 2015, 14: 1627-1646

41 Luo H J, Guo Z W, Dong W, et al. LDB: localization with directional beacons for sparse 3D underwater acoustic sensor networks. J Netw, 2010, 5: 28-38

42 Pandey O J, Sharan R, Hegde R M. Localization in wireless sensor networks using visible light in non-line of sight conditions. Wirel Personal Commun, 2017, 6: 1-21

43 科技日报. 国产高精度 “超短基线定位系统” 打破国外垄断, 2014

44 光明日报.“蛟龙”号用上国产高精度超短基线定位系统, 2014

45 中国海洋报. 2015 年度中国海洋十大科技进展发布, 2016

46 刘健, 徐会希. 潜龙一号: 深海里的中国龙. 科技纵览, 2014, 6: 92-93

47 Ji D X, Liu J, Chen X Z, et al. Fast and accurate AUV positioning using acoustic beacons of LBL system. Tech Acoust, 2009, 28: 476-479 [冀大雄, 刘健, 陈孝桢, 等. 基于 LBL 声信标的 AUV 快速精确定位. 声学技术, 2009, 28: 476-479]

48 Su R Y, Venkatesan R, Li C. A new node coordination scheme for data gathering in underwater acoustic sensor networks using autonomous underwater vehicle. In: Proceedings of Wireless Communications and Networking Conference (WCNC), Shanghai, 2013. 4370-4374

49 Hollinger G A, Choudhary S, Qarabaqi P, et al. Underwater data collection using robotic sensor networks. IEEE J Sel Areas Commun, 2012, 30: 899-911

50 Fazel F, Stojanovic M. Resource allocation for hierarchical underwater sensor networks. In: Proceedings of Information Theory and Applications Workshop (ITA), San Diego, 2013. 1-10

51 Hajihoseini G A, Shahbazian R, Ghorashi S A. Decentralized consensus based target localization in wireless sensor networks. Wirel Pers Commun, 2017, 97: 3587-3599

52 Han G, Jiang J, Chao J, et al. Path planning for a group of mobile anchor nodes based on regular triangles in wireless sensor networks. Neurocomputing, 2017, 270: 198-208

53 Sharma G, Kumar A. Dynamic range normal bisector localization algorithm for wireless sensor networks. Wirel Pers Commun, 2017, 8: 1-21

54 Zhang X R, Xiong W L, Xu B G. A computationally efficient received signal strength based localization algorithm in closed-form for wireless sensor network. Neural Process Lett, 2017, 3: 1-15

55 Kumar G, Rai M K, Saha R. Securing range free localization against wormhole attack using distance estimation and maximum likelihood estimation in wireless sensor networks. J Netw Comput Appl, 2017, 99: 10-16

56 Liu L, Du J, Guo D. A clustering approach for error beacon filtering in underwater wireless sensor networks. Int J Distrib Sens Netw, 2016, 12: 1-10

57 Chandrasekhar V, Seah W. An area localization scheme for underwater sensor networks. In: Proceedings of OCEANS, Singapore, 2007. 1-8

58 Aziz M, Tayarani-N M H, Meybodi M R. A two-objective memetic approach for the node localization problem in wireless sensor networks. Genetic Programm Evol Mach, 2016, 17: 321-358 
59 Cheng X Z, Shu H N, Liang Q L, et al. Silent positioning in underwater acoustic sensor networks. IEEE Trans Veh Technol, 2008, 57: 1756-1766

60 Shang F, Jiang Y, Xiong A, et al. A node localization algorithm based on multi-granularity regional division and the lagrange multiplier method in wireless sensor networks. Sensors, 2016, 16: 1934

61 Carroll P, Mahmood K, Zhou S, et al. On-demand asynchronous localization for underwater sensor networks. IEEE Trans Signal Process, 2014, 62: 3337-3348

62 Teymorian A Y, Cheng W, Ma L, et al. 3D underwater sensor network localization. IEEE Trans Mobile Comput, 2009, 8: 1610-1621

63 Chizari H, Poston T, Abd Razak S, et al. Local coverage measurement algorithm in GPS-free wireless sensor networks. Ad Hoc Netw, 2014, 23: 1-17

64 Zhou Y, He J H, Chen K, et al. An area localization scheme for large scale underwater wireless sensor networks. In: Proceedings of the 2009 WRI International Conference on Communications and Mobile Computing. Washington: IEEE Computer Society, 2009. 543-547

65 Liu W, Wang D, Jiang H, et al. An approximate convex decomposition protocol for wireless sensor network localization in arbitrary-shaped fields. IEEE Trans Parall Distrib Syst, 2015, 26: 3264-3274

66 Erol M, Vieira L M, Gerla M. AUV-aided localization for underwater sensor networks. In: Proceedings of International Conference on Wireless Algorithms, Systems and Applications (WASA 2007), Chicago, 2007. 44-54

67 Luo H J, Zhao Y Y, Guo Z W, et al. UDB: using directional beacons for localization in underwater sensor networks. In: Proceedings of International Conference on Parallel and Distributed Systems, Melbourne, 2008. 551-558

68 Luo H J, Guo Z W, Dong W, et al. LDB: localization with directional beacons for sparse 3D underwater acoustic sensor networks. J Netw, 2010, 5: 28-38

69 Rehman Y A U, Tariq M, Khan O U. Improved object localization using accurate distance estimation in wireless multimedia sensor networks. PLoS ONE, 2015, 10: e0141558

70 You K, Yang W, Han R. The video collaborative localization of a Miner's lamp based on wireless multimedia sensor networks for underground coal mines. Sensors, 2015, 15: 25103-25122

71 Han G, Zhang C, Liu T, et al. MANCL: a multi-anchor nodes collaborative localization algorithm for underwater acoustic sensor networks. Wirel Commun Mob Comput, 2016, 16: 682-702

72 Zhao R Q, Shen X H, Gao J J, et al. Localization in underwater acoustic networks based on double rates. In: Proceedings of OCEANS, Hampton Roads, 2012. 1-4

73 Zhu Z W, Guan W B, Liu L F, et al. A multi-hop localization algorithm in underwater wireless sensor networks. In: Proceedings of the 6th International Conference on Wireless Communications and Signal Processing (WCSP), Hefei, 2014. $1-6$

74 Liu J, Wang Z H, Zuba M, et al. JSL: joint time synchronization and localization design with stratification compensation in mobile underwater sensor networks. In: Proceedings of the 9th Annual IEEE Communications Society Conference on Sensor, Mesh and Ad Hoc Communications and Networks (SECON), Seoul, 2012. 317-325

75 Liu J, Wang Z, Cui J H, et al. A joint time synchronization and localization design for mobile underwater sensor networks. IEEE Trans Mobile Comput, 2016, 15: 530-543

76 Diamant R, Lampe L. Underwater localization with time-synchronization and propagation speed uncertainties. IEEE Trans Mobile Comput, 2013, 12: 1257-1269

77 Yi J W, Mirza D, Schurgers C, et al. Joint time synchronization and tracking for mobile underwater systems. Ad Hoc Netw, 2013, 34: 1-8

78 Yi J W, Mirza D, Kastner R, et al. ToA-TS: time of arrival based joint time synchronization and tracking for mobile underwater systems. Ad Hoc Netw, 2015, 211-223

79 Xia Y, Wang Y, Ma X, et al. Joint time synchronization and localization for underwater acoustic sensor networks. In: Proceedings of the 10th International Conference on Underwater Networks \& Systems. New York: ACM, 2015

80 He C F, Wang Y Y, Chen C, et al. Underwater acoustic localization with uncertainties in propagation speed and time synchronization. In: Proceedings of MTS/IEEE Monterey OCEANS, Monterey, 2016. 1-5

81 Ramezani H, Leus G. Localization packet scheduling for underwater acoustic sensor networks. IEEE J Sel Areas Commun, 2015, 33: 1345-1356

82 Ramezani H, Fazel F, Stojanovic M, et al. Collision tolerant and collision free packet scheduling for underwater acoustic 
localization. IEEE Trans Wireless Commun, 2015, 14: 2584-2595

83 Zhang S L, Zhang Q, Liu M Q, et al. A multi-hop reverse localization scheme for underwater wireless sensor networks. In: Proceedings of the 32nd Chinese Control Conference, Xi'an, 2013. 7383-7388

84 Liu B, Chen H, Zhong Z, et al. Asymmetrical round trip based synchronization-free localization in large-scale underwater sensor networks. IEEE Trans Wirel Commun, 2010, 9: 3532-3542

85 Han Y F, Zheng Y R, Sun D J. Measurement error impact on node localization of large scale underwater sensor networks. In: Proceedings of IEEE 82nd Vehicular Technology Conference (VTC2015-Fall), Boston, 2015

86 Guo Z, Guo Y, Hong F, et al. Perpendicular intersection: locating wireless sensors with mobile beacon. IEEE Trans Veh Technol, 2010, 59: 3501-3509

\title{
Developing status and some cutting-edge issues of underwater sensor network localization technology
}

\author{
Dajun SUN, Cuie ZHENG*, Hongyu CUI, Jucheng ZHANG \& Yunfeng HAN \\ College of Underwater Acoustic Engineering, Harbin Engineering University, Harbin 150001, China \\ * Corresponding author. E-mail: zhengcuie@hrbeu.edu.cn
}

\begin{abstract}
Owing to the complicated marine environment, a vast majority of terrestrial wireless sensor network technologies cannot be used directly in underwater sensor networks, which should be studied in combination with the special marine environment. In the last decade, there has been great progress in underwater sensor network localization technology. This paper summarizes the developing status first, and then puts forward some cutting-edge issues about underwater sensor network localization technology. Finally, this paper forecasts some practical questions expected to be solved in the next few years about underwater sensor network node localization technology.
\end{abstract}

Keywords underwater wireless sensor networks, localization, communication, network positioning node, underwater signal propagation

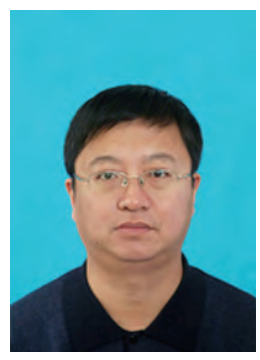

Dajun SUN received the B.S., M.S., and Ph.D. degrees in underwater acoustic engineering from Harbin Engineering University, Harbin, China, in 1994 1996, and 1999, respectively. He worked at Harbin Engineering University from 1996 and studied at the National University of Singapore as a visiting scholar from 2001 to 2002. He became a professor in 2007. His research focuses on sonar system design and application research, including long/short-baseline localization sonar, Doppler velocity logs, communication sonar, submerged buoy systems, and imaging sonar.

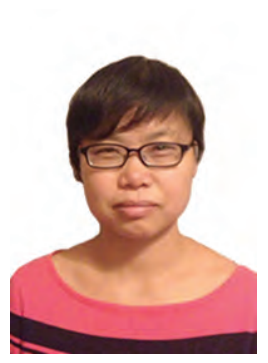

Cuie ZHENG received the B.S. and Ph.D. degrees in underwater acoustic engineering from Harbin Engineering University, Harbin, China, in 2004 and 2008, respectively. She worked at the University of Victoria as a visiting scholar from 2016 to 2017. Since 2006, she has been with the College of Underwater Acoustic Engineering at Harbin Engineering University, where she is currently an associate professor. Her research interests lie in algorithms and applications of underwater acoustic localization and navigation.

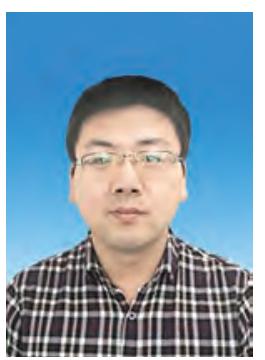

Jucheng ZHANG received the B.S. and Ph.D. degrees in underwater acoustic engineering from Harbin Engineering University, Harbin, China, in 2009 and 2014, respectively. Since 2014, he has been with the College of Underwater Acoustic Engineering at Harbin Engineering University, where he is currently an associate professor. His research interests lie in algorithms and applications of underwater acoustic localization and navigation. 